\title{
Hospital Readmissions of Discharged Patients with COVID-19
}

This article was published in the following Dove Press journal:

International Journal of General Medicine

\author{
Lina Marcela Parra $\mathbb{D}^{\prime}$ \\ Mireia Cantero $\mathbb{D}^{\prime}$ \\ Ignacio Morrás ${ }^{2}$ \\ Alberto Vallejo' \\ Itziar Diego ${ }^{2}$ \\ Elena Jiménez-Tejero' \\ Elena Múñez ${ }^{3}$ \\ Ángel Asensio' \\ Ana Fermández-Cruz $\mathbb{D}^{3,4}$ \\ Antonio Ramos-Martinez $\mathbb{D D}^{3,4}$ \\ On behalf of Puerta de Hierro \\ Hospital Admission Study \\ Group
}

'Servicio de Medicina Preventiva, HU Puerta de Hierro-Majadahonda, Madrid, Spain; ${ }^{2}$ Servicio de Medicina Interna, HU Puerta de Hierro-Majadahonda, Madrid, Spain; ${ }^{3}$ Servicio de Medicina Interna, HU Puerta de Hierro-Majadahonda, Unidad de Enfermedades Infecciosas, UAM, Madrid, Spain; ${ }^{4}$ Instituto Investigación Sanitaria Puerta de Hierro - Segovia de Arana (IDIPHSA), Madrid, Spain
Correspondence: Antonio Ramos-Martinez Servicio de Medicina Interna, HU Puerta de Hierro-Majadahonda, Unidad de Enfermedades Infecciosas, Calle Joaquín Rodrigo 2, Majadahonda, Madrid 28222, Spain

Tel +3463821। 120

$\mathrm{Fax}+34911916807$

Email aramos2202@gmail.com
Objective: To analyse the rate of occurrence and the clinical variables associated with readmission of patients who had previously been discharged after admission for COVID-19. Setting: University hospital in Madrid (Spain).

Participants: Sixty-one patients (74\% male) who presented COVID-19 were readmitted during the 3 weeks after discharge from hospital.

Interventions: Nested case-control study paired (1:1 ratio) by age, sex and period of admission.

Outcome Measures: Rate of readmission rate of patients discharged after suffering COVID-19 and identification of the clinical variables associated with it.

Results: Out of 1368 patients who were discharged during the study period, 61 patients (4.4\%) were readmitted. Immunocompromised patients $(\mathrm{N}=10.2 \%)$ were at increased risk for readmission $(\mathrm{p}=0.04)$. There was also a trend towards a higher probability of readmission in hypertensive patients $(\mathrm{p}=0.07)$. Cases had had a shorter hospital stay and a higher prevalence of fever during the 48 hours prior to discharge. There were no significant differences in oxygen levels measured at admission and discharge by pulse oximetry intra-subject or between the groups. Neutrophil-to-lymphocyte ratio at hospital admission tended to be higher in cases than in controls $(\mathrm{p}=0.06)$. Neither glucocorticoids nor anticoagulants prescribed at hospital discharge were associated with a lower readmission rate. Patients who were readmitted due to a thrombotic event ( 8 patients, $13.1 \%$ ) presented a higher level of D-dimer at discharge of initial admission.

Conclusion: The rate of readmission after discharge from hospital for COVID-19 was low. Immunocompromised patients and those presenting with fever during the 48 hours prior to discharge were at greater risk of readmission to hospital.

Keywords: coronavirus, pneumonia, viral, patient readmission, pulmonary embolism, heparin

\section{Plain Language Summary}

COVID-19 is a disease that occurs in epidemic outbreaks and may require a large number of hospital beds to be available for a short period of time. As it is a new disease, its clinical evolution is not well studied. It is known that there is an "inflammatory phase" after the viral infection that can lead to worsening caused by the involvement of several organs (lung, brain, heart, digestive tract) and due to various mechanisms (infection, inflammation, formation of thrombi within the blood vessels). Knowing the characteristics of patients who worsen after discharge from the hospital can help to better decide the time of discharge and makes a closer follow-up once the patient goes home. We have found that patients with low defenses (immunosuppressed patients) and those who have fever during the 48 hours prior to discharge are at increased risk of readmission to the hospital. We believe that the sharing of 
these findings could make the clinical management of patients with COVID-19 more effective and appropriate.

\section{Introduction}

The dramatic effects of the COVID-19 pandemic on the population have overwhelmed many health institutions around the world. During several weeks, the demand for hospital beds has exceeded the capacity to admit patients. ${ }^{1}$ In addition, the high hospital attendance rate has reduced the capability to treat other serious diseases such as neoplasms or cardiovascular diseases. ${ }^{2,3}$ Therefore, making the most efficient use of hospital beds should be an imperative objective during outbreaks of this nature.

Clear guidelines on when to discharge patients admitted with this infection based on their clinical evolution have not yet been established. ${ }^{4}$ Therefore, physicians may act too cautiously, protracting hospital stay unnecessarily, or conversely, they may be too bold in discharging patients quickly, potentially worsening their prognosis. Although the study of readmitted patients with COVID19 is a very relevant problem, to date very little attention has been devoted to its different clinical aspects. ${ }^{5-7}$ Knowledge of the characteristics of patients who are most at risk of being readmitted to the hospital in the following weeks could help to better decide when to discharge and to select patients who need closer clinical follow-up after discharge.

Therefore, we planned a study to find out the rate of occurrence and the clinical variables associated with readmission of patients discharged after suffering from COVID-19

\section{Methods}

\section{Setting}

The study was conducted in a 613-bed tertiary care university hospital in Madrid (Spain). The first case of SARSCoV-2 infection was confirmed on 25 February, 2020 in the Community of Madrid. Since then, this region accounts for 68,696 confirmed cases, 42,928 inpatients and 14,671 deaths, being the most affected area in Spain. In our hospital, the first case was identified on 27 February, and there have been nearly 1500 in-patients. The highest count of admitted patients was 626 , which was reached on 30 March 30.

\section{Study Population}

We studied a retrospective cohort of patients with laboratory-confirmed SARS-CoV-2 infection who were admitted and subsequently discharged alive. The study period extended between 26 February and 20 April 2020. SARSCoV-2 was detected by a real-time PCR assay targeting E-gene, RdRP-gene and N-gene, performed with the protocol reported by the WHO. ${ }^{8}$

\section{Case-Control Study}

We conducted a nested case-control study matched (ratio $1: 1)$ by age, sex and period of admission. Two periods were defined based on the incidence of admission cases. The first covered from 26 February to 26 March, the period of highest cumulative incidence of admission cases, and the second from 27 March to 20 April.

A case-patient was defined as a patient with confirmed SARS-CoV-2 infection who had been readmitted within 3 weeks of discharge and clinical presentation of readmission was related to the infection or its treatment. Patients who were discharged but not readmitted were considered as controls, after excluding death during that period.

The medical data included parameters such as obesity, cancer, diabetes, chronic obstructive pulmonary disease (COPD), immunosuppression and hypertension. Obesity was defined as a body mass index greater than $30 \mathrm{~kg} / \mathrm{m}^{2}$. Immunocompromised patients included those with solid organ or hematopoietic stem cell transplantation, HIV infection, or who were previously treated more than 3 weeks with glucocorticoids (equivalent dose of prednisone $\geq 15 \mathrm{mg} /$ day) or immunosuppressive drugs. Symptoms (including their length), physical examination findings and laboratory measurements were extracted from the electronic medical record. Analytical data (hemogram, basic biochemistry, lactate dehydrogenase, C-reactive protein, D-dimer and ferritin) at admission and discharge were recorded. To evaluate the radiological evolution, we consider the corresponding radiologist report. No specific discharge criteria were established. The usual criteria in community pneumonia were used, such as disappearance of fever, improvement of the general condition and respiratory failure. Outcomes included death and hospital discharge.

Clinical presentations related to the infection or complications related to treatment for SARS-CoV-2 infection were registered as reasons for admission, including respiratory manifestations, venous or arterial thrombosis, exacerbation of chronic disease, organ failure, bacterial superinfection.

\section{Statistical Analysis}

The chi-squared test and Fisher's exact test were used to compare categorical variables, and continuous variables by 
Table I Clinical Characteristics of All COVID-19 Patients Admitted During the Study Period

\begin{tabular}{|l|l|l|l|}
\hline Characteristics & $\begin{array}{l}\text { Patient with a Single Admission } \\
\text { (n=1307) }\end{array}$ & $\begin{array}{l}\text { Readmitted Patients } \\
\text { (n=6 I) }\end{array}$ & p \\
\hline Age (median, IQR) & $64(54-75)$ & $67(59-76)$ & 0.14 \\
Male (n, \%) & $827(63.3)$ & $45(73.8)$ & 0.10 \\
Length of symptoms (median, IQR) & $7(4-10)$ & $6(3-10)$ & 0.43 \\
Admission to ICU (n, \%) & $93(7.1)$ & $3(4.9)$ & 0.51 \\
Non-survivors (n, \%) & $163(12.5)$ & $9(14.7)^{\mathrm{a}}$ & 0.61 \\
\hline
\end{tabular}

Note: ${ }^{\text {a }}$ atients who died during hospital readmission.

Abbreviations: IQR, interquartile range; ICU, intensive care unit.

Table 2 Cause of Hospital Admission in Cases (First Admission) and Controls

\begin{tabular}{|l|l|l|l|}
\hline Condition & Cases $(\mathbf{n}=6 \mathbf{I})$ & Controls $(\mathbf{n = 6} \mathbf{I})$ & $\mathbf{p}$ \\
\hline Pneumonia & $53(86.9)$ & $59(96.7)$ & 0.05 \\
Heart failure & $2(3.3)$ & $\mathrm{I}(1.64)$ & 0.55 \\
Bacterial superinfection & $5(8.2)$ & $4(6.7)^{\mathrm{a}}$ & 0.75 \\
Acute kidney failure & $\mathrm{I}(1.6)$ & $0(0.0)$ & 0.32 \\
\hline
\end{tabular}

Note: ${ }^{a}$ In three patients there was lung co-infection.

Student's $t$-test and the Mann-Whitney $U$-Test. Univariate and multivariate logistic regression was undertaken with Stata 13.0 software (StataCorp, College Station, US). All tests of significance were two-tailed, and values of $p<0.05$ were considered statistically significant.

\section{Data Statement}

The information contained in the database can be obtained through the corresponding author. There are legal difficulties for direct access from the journal.

\section{Patient and Public Involvement Statement}

Taken into account the type of research, it was not appropriate to involve patients nor the public in the design, or conduct, or reporting, or dissemination plans of our research.

\section{Results}

Out of 1368 patients who were discharged during the study period, 61 patients $(4.4 \%)$ were readmitted (Table 1, Figure 1S (Supplementary Material). The median time from discharge to readmission was 6 days (IQR 3-10). One hundred and sixty-three patients (12.5\%) died during the first admission.

\section{Clinical Characteristics of the Patients Who are Readmitted}

Table 2 shows the causes for admission in the cases (first admission) and the controls and Table 3 the cause of hospital admission and readmission in the cases. Admission due to pneumonia tended to be more frequent among controls than among cases.

Table 4 displays demographic and clinical characteristics of cases and controls. Immunocompromised patients were at increased risk for readmission. There was also a trend towards a higher probability of readmission in hypertensive patients $(\mathrm{p}=0.07)$. Cases had had a shorter hospital stay and a higher prevalence of fever during the 48 hours prior to

Table 3 Cause of Hospital Admission and Readmission in Cases

\begin{tabular}{|c|c|c|}
\hline Condition & $\begin{array}{l}\text { Initial } \\
\text { Admission } \\
(n=6 I)\end{array}$ & $\begin{array}{l}\text { Readmission } \\
(n=6 I)\end{array}$ \\
\hline $\begin{array}{l}\text { Pneumonia } \\
\text { Pulmonary thromboembolism } \\
\text { Heart failure } \\
\text { Bacterial infection } \\
\text { Acute kidney failure } \\
\text { Deep vein thrombosis } \\
\text { Lower limb arterial } \\
\text { thrombosis } \\
\text { Myocardial acute infarction } \\
\text { Severe bleeding } \\
\text { Diabetes, hyperglycaemic } \\
\text { hyperosmolar state } \\
\text { Generalized oedema } \\
\text { Threatened miscarriage }\end{array}$ & $\begin{array}{l}53(86.9) \\
2(3.3) \\
5(8.2) \\
1(1.6)\end{array}$ & $\begin{array}{l}34(55.7)^{\mathrm{a}} \\
8(13.1)^{\mathrm{a}} \\
6(9.8) \\
4(6.6) \\
2(3.3) \\
\text { I }(1.6) \\
\text { I }(1.6) \\
I(I .6) \\
I(I .6) \\
\text { I }(1.6) \\
I(I .6) \\
\text { I }(1.6)\end{array}$ \\
\hline
\end{tabular}

Note: ${ }^{a} p<0.001$. 
Table 4 Demographic and Clinical Characteristics in Cases and Controls

\begin{tabular}{|c|c|c|c|}
\hline Characteristics & Cases $(n=61)$ & Controls $(n=6 I)$ & $\mathbf{p}$ \\
\hline Age (median, IQR) & $67(59-76)$ & $66(57-76)$ & 0.68 \\
\hline Male (n, \%) & $45(73.8)$ & $45(73.8)$ & 1.00 \\
\hline \multicolumn{4}{|l|}{ Comorbidity } \\
\hline Obesity & $6(9.8)$ & $5(8.2)$ & 0.75 \\
\hline Diabetes mellitus & $14(22.9)$ & $10(16.4)$ & 0.36 \\
\hline Hypertension & $34(55.4)$ & $24(39.3)$ & 0.07 \\
\hline Cardiovascular disease & $16(26.2)$ & $12(19.7)$ & 0.39 \\
\hline COPD & $12(19,7)$ & $12(19.7)$ & 1.00 \\
\hline Neoplasia & $12(19.7)$ & $12(19.7)$ & 1.00 \\
\hline Immunosuppression $^{\mathrm{a}}$ & $10(16.4)$ & $3(4.9)$ & 0.04 \\
\hline \multicolumn{4}{|l|}{ Initial admission } \\
\hline Length of symptoms (median, IQR) & $6(3-10)$ & $7(4-9)$ & 0.52 \\
\hline Admission to ICU (n, \%) & $3(4.9)$ & $5(8.2)$ & 0.72 \\
\hline Total hospital stay (median, IQR) & $6(4-14)$ & $9(6-14)$ & 0.02 \\
\hline Pneumonia on admission $(\mathrm{n}, \%)$ & $53(86.9)$ & $59(96.7)$ & 0.05 \\
\hline Oxygen saturation $(\%, \text { mean, SD })^{b}$ & $94.9(2.7)$ & $94.7(2.0)$ & 0.68 \\
\hline \multicolumn{4}{|l|}{ Discharge } \\
\hline Fever 48 hours at discharge $(n, \%)$ & II (18.0) & $4(6.6)$ & $<0.001$ \\
\hline Afebrile at discharge (days, median, IQR) & $5(3-10)$ & $7(5-11)$ & 0.03 \\
\hline Oxygen saturation $(\%, \text { mean, } S \mathrm{SD})^{\mathrm{b}}$ & $93.7(12.4)$ & $94.9(2.2)$ & 0.46 \\
\hline \multicolumn{4}{|l|}{ Radiological evolution (n, \%) } \\
\hline No change & $21(42.9)$ & $18(38.3)$ & 0.31 \\
\hline Worsening & $16(32.7)$ & II (23.4) & \\
\hline Improving & $12(24.4)$ & $18(38.3)$ & \\
\hline Glucocorticoid treatment at discharge $(n, \%)$ & $30(49.2)$ & $26(42.62)$ & 0.47 \\
\hline Anticoagulants at discharge ${ }^{c}(\mathrm{n}, \%)$ & $16(26.2)$ & $17(28.9)$ & 0.84 \\
\hline
\end{tabular}

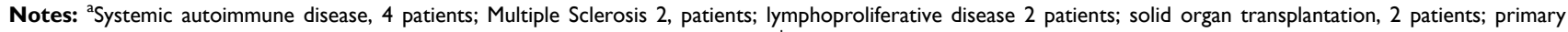

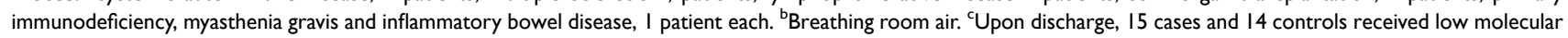
weight heparin in prophylactic doses.

Abbreviations: IQR, interquartile range; SD, standard deviation; COPD, chronic obstructive pulmonary disease.

discharge. There were no significant differences in oxygen levels measured at admission and discharge by pulse oximetry intra-subject or between the groups. Neither glucocorticoids nor anticoagulants prescribed at hospital discharge were associated with a lower readmission rate (Table 4). Seventeen cases and 16 controls received anticoagulant treatment. Anticoagulant treatment consisted of low molecular weight heparin (LMWH) at prophylactic doses in $88.2 \%$ of cases and $87.5 \%$ of controls, respectively.

No difference in analytical data at admission or discharge was evident between the two groups (Table 5). Neutrophil-to-lymphocyte ratio at hospital admission tended to be higher in cases than in controls $(\mathrm{p}=0.06)$. Multivariate analysis of the variables most related to hospital readmission in patients with COVID-19 are shown in Tables 1S (Supplementary Material).
There were 33 patients who had repeat PCR at the time of readmission, 16 of whom (45.5\%) were positive, resulting in a reduced number of positive patients compared to the index admission. There was a trend towards a higher incidence of pneumonia in patients with positive PCR on readmission (11 patients, 68.7\%) than in patients with negative PCR (6 patients, $35.3 \%, \mathrm{p}=0.055$ ). Other causes of readmission in patients with positive PCR were pulmonary thromboembolism (three patients, 18.7\%), systemic arterial ischemia (one patient, 6.2\%) and delivery (one patient, 6.2\%). Other causes of readmission in patients with negative PCR were pulmonary thromboembolism (three patients, $17.6 \%$ ), heart failure (three patients, 17.6\%), bacterial superinfection (three patients, 17.6\%), and decompensation of the underlying disease (two patients, 11.7\%). The comparison of other clinical and 
Table 5 First and Last Laboratory Analysis Results of Patients with COVID-19

\begin{tabular}{|c|c|c|c|}
\hline & Cases & Controls & $\mathbf{p}$ \\
\hline \multicolumn{4}{|l|}{ At initial admission (median, IQR) } \\
\hline C-reactive protein, $\mathrm{mg} / \mathrm{L}$ & $34.5(15.8-100.2)$ & $37.8(8.7-70.0)$ & 0.28 \\
\hline D-Dimer, ng/mL & $1.08(0.48-1.94)$ & $0.65(0.36-1.54)$ & 0.09 \\
\hline Lactate dehydrogenase, units/L & $266.5(216-322)$ & $251.0(213-323)$ & 0.62 \\
\hline Ferritin, $\mathrm{ng} / \mathrm{mL}$ & $756(302-208 I)$ & $603(370-869)$ & 0.55 \\
\hline Lymphocyte count, cells/ $\mu \mathrm{L}$ & $970(670-1460)$ & $1090(870-1430)$ & 0.31 \\
\hline Neutrophil count, cells $/ \mu \mathrm{L}$ & $4390(3070-7300)$ & $3960(2770-6110)$ & 0.30 \\
\hline Neutrophil-to-lymphocyte ratio & $4.32(2.82-7.40)$ & $3.23(2.30-5.31)$ & 0.06 \\
\hline \multicolumn{4}{|l|}{ Pre-discharge (median, IQR) } \\
\hline C-reactive protein, mg/L & I $5.7(5.2-49.5)$ & I5.3 (4.0-35.2) & 0.45 \\
\hline D-Dimer, ng/mL & $0.89(9.38-1.44)$ & $0.60(0.39-1.37)$ & 0.41 \\
\hline Lactate dehydrogenase, units/L & $225(178-278)$ & $24 I(192-286)$ & 0.50 \\
\hline Ferritin, $\mathrm{ng} / \mathrm{mL}$ & $670(416-1434)$ & $469(308-876)$ & 0.11 \\
\hline 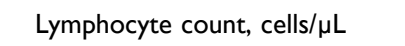 & $1070(600-1700)$ & $1360(900-1620)$ & 0.09 \\
\hline Neutrophil count, cells $/ \mu \mathrm{L}$ & $3820(2100-5700)$ & $3940(2865-5480)$ & 0.53 \\
\hline Neutrophil-to-lymphocyte ratio & $3.13(1.90-8.43)$ & $3.01(1.74-5.68)$ & 0.35 \\
\hline
\end{tabular}

Abbreviation: IQR, interquartile range.

analytical variables between both groups is shown in Tables 2S and 3S (Supplementary Material). The motive for readmission in 10 patients $(16.4 \%)$ was a thrombotic event in venous or arterial territory. Among them, seven patients had no anticoagulant medication recommended at discharge $(\mathrm{p}=0.07)$. All cases that presented pulmonary thromboembolism or deep vein thrombosis were diagnosed during the second admission ( $\mathrm{p}=0.001)$. Readmitted patients due to pulmonary thromboembolism (eight patients, 13.1\%) presented a higher pre-discharge D-Dimer level [median; interquartile range (IQR) 1.41; 1.11-2.43] than the rest of the patients (median 0.65; IQR: $0.34-1.23 ; \mathrm{p}=0.011$ ).

\section{Discussion}

The readmission rate shown in this study may be considered as moderate, which suggests that discharge decisions were frequently appropriate and the prognosis for most patients was favourable. Immunocompromised patients and those who presented fever within 48 hours prior to discharge were at increased risk of readmission.

These results are similar to those of other published studies, with a readmission rate during the first weeks between $2 \%$ and $4 \%{ }^{6,7}$ The vast majority of COVID-19 patients who are discharged are not readmitted, suggesting that they are not discharged hastily. In addition, the readmission rate of COVID-19 patients appears to be lower than that observed in conventional patients treated in internal medicine wards. This fact may be related to the more advanced age and relevant comorbidity in the latter. ${ }^{9}$ In any case, further research would be desirable to analyse how to adjust the hospital stay even further without significantly increasing readmissions.

\section{Clinical Characteristics of the Patients Who are Readmitted}

Our immunocompromised patients were at increased risk for readmission. The effect of immunosuppression on the clinical course of SARS-Cov-2 infection is not well known. Long-term use of glucocorticoids can cause atypical clinical presentation with a longer incubation period, which could reduce the clinical suspicion of this infection. ${ }^{10}$ Likewise, mild cases have been described in relation to the mitigation of cytokine storm by the previous use of immunosuppressive drugs. ${ }^{11}$ In fact, some immunocompromised patients are receiving treatment with interleukin inhibitors such as tocilizumab or anakinra, which are drugs that have been successfully used in patients with COVID-19. On the other hand, it has been observed that transplanted patients have a clearly worse prognosis than other types of patients. ${ }^{12}$ These discordant findings may be due to the different effects of each type of immunosuppression on innate and acquired immunity and the uncertainty of the role of viral replication in the prognosis of the disease. ${ }^{12-14}$ Until the prognosis and course of immunosuppressed patients with COVID-19 is better 
understood, close monitoring is desirable to promptly detect the eventual occurrence of clinical worsening.

Patients with hypertension in our series showed a trend towards a more frequent readmission $(\mathrm{p}=0.07)$. Other studies have reported a higher risk of readmission in patients with hypertension, which could be related to the more severe disease of COVID-19 in hypertensive patients. ${ }^{7}$ Our study did not show an increased risk of readmission in COPD patients, which, according to Sulaiman et $\mathrm{al}^{7}{ }^{7}$ seems to be expected from the hypoxemia associated with respiratory infections in these patients. Patients who present with fever during the 48 hours prior to discharge are at increased risk of readmission and should be followed closely in order to detect relevant problems in their clinical evolution and to be able to receive an earlier treatment. Differences in age between cases and controls were not analysed, due to age-adjusted case-control matching, but the previous study showed no difference in age in terms of risk of readmission. ${ }^{7}$ Of note, analytical alterations (LDH, C-reactive protein, neutrophil-to-lymphocyte ratio, and $\mathrm{D}$-dimer) were not useful in predicting readmission. There was a trend towards higher D-dimer and neutrophil-to-lymphocyte ratios on admission and lower lymphocyte counts on discharge in patients who were readmitted (Table 5). This result has also been reported by other authors and reinforces the belief that clinical variables (such as fever or respiratory failure) are more important than analytic ones (such as the degree of lymphopenia or the plasma level of C-reactive protein) for decisionmaking in this disease. ${ }^{7}$

\section{Description of the Cause for Admission at Initial Admission and Readmission}

The immobilization due to asthenia and malaise, hypoxia, coagulopathy and endothelial viral damage that characterize COVID-19 predispose patients to venous thromboembolic disease (VTE). ${ }^{15,16}$ Nevertheless, cases of VTE usually appear progressively during the 4 weeks after the first arrival in hospital, which may explain why it is a more frequent cause of admission in the second than in the first admission (Table $3)^{16}$ The use of prophylactic LMWH in our institution increased as we became more aware of the risk of VTE. However, the prophylactic strategy with LMWH has not been successful in all cases. ${ }^{17}$ Patients who were readmitted for vascular thrombotic problems tended to have been discharged from the initial admission without anticoagulant medication $(p=0.07)$. The concentration at discharge of the first admission of D-dimer was higher in patients who were readmitted for pulmonary embolism. Although the relationship between D-dimer and VTE risk in patients with COVID-19 has been established previously, a recommendation for VTE prophylaxis based on a specific plasma D-dimer concentration of the patient at discharge has not yet been carried out. Closer clinical surveillance and prescription of prophylactic heparin could reduce the incidence of severe cases of TVE in patients at greatest risk. $^{18}$

Moreover, treatment of patients during initial admission with intravenous fluids, glucocorticoids and anticoagulants may cause worsening of chronic diseases such as heart failure or diabetes and end up causing readmissions. In addition, uncontrolled diabetic patients may present impaired adaptive immune response due to an inadequate $\mathrm{CD} 4+$ T-cell response. ${ }^{19}$ The careful use of medication and optimal medical care is a clear objective in the management of these patients.

\section{Strengths and Limitations}

The strength of the study is based on the determination of the rate of readmissions and their causes and its case-control design which has allowed the identification of the clinical profile of patients who readmit after suffering COVID-19. The results of the study are useful for deciding when to discharge these patients. This study has several limitations that should be highlighted. First, the small sample size may have prevented the identification of differences in some variables. Secondly, it is a single-centre study having its own diagnostic and therapeutic peculiarities. Thirdly, we cannot completely exclude that some of the control patients had been admitted to a private hospital, whose information is not collected in the public health informatics system. However, this is not likely to have occurred. And finally, it should be noted that the demand for hospital admissions and the learning curve of the disease has changed over the course of the epidemic, which may have had a varying impact on patient discharge.

In summary, we report a low rate of readmission after discharge from hospital for COVID-19. Immunocompromised patients and those presenting with fever during the 48 hours prior to discharge are at greater risk of readmission to hospital. Given the possibility of further outbreaks of the disease, further research should be encouraged to refine the risk factors for hospital readmission that could help to safely discharge these patients.

\section{Ethical Statement}

The study was approved by the Institutional Review Board (CEIm) at Hospital Universitario Puerta de HierroMajadahonda, and a waiver for the informed consent was 
granted (PI_154_2020). The study complied with the provisions in EU and Spanish legislation on data protection and the Declaration of Helsinki 2013.

\section{Acknowledgments}

Puerta de Hierro COVID19 Hospital Readmission Study Group Collaborators were as follows: Múñez Rubio, Elena; Malo de Molina Ruiz, Rosa; Pintos Pascual, Ilduara; Callejas Díaz, Alejandro; Díaz De Santiago, Alberto; Fernández Cruz, Ana; Ramos Martínez, Antonio; De La Fuente Moral, Sara; Valle Falcones, Manuel; Muñoz Serrano, Alejandro; Calderón Parra, Jorge; Ángel-Moreno Maroto, Alfonso; Baños Pérez, Isolina; Máinez Sáiz, María Carmen; Moreno-Torres Concha, Víctor; Romero Pizarro, Yolanda; Aguilar Pérez, Miriam; Díaz Nuevo, Guadalupe Gema; García Fadul, Christian; Jara Chinarro, Beatriz; Laporta Hernández, Rosalía; Lázaro Carrasco de la Fuente, María Teresa; López García Gallo, Cristina María; Fernández, Carmen; Vargas Núñez, Juan Antonio; Ussetti Gil, Piedad; CuervasMons Martínez, Valentín.

\section{Author Contributions}

All authors made a significant contribution to the work reported, whether that is in the conception, study design, execution, acquisition of data, analysis and interpretation, or in all these areas; took part in drafting, revising or critically reviewing the article; gave final approval of the version to be published; have agreed on the journal to which the article has been submitted; and agree to be accountable for all aspects of the work.

\section{Funding}

This study did not receive any funding.

\section{Disclosure}

Ana Fernández Cruz reports advisory for Gilead and educational activity for MSD, outside the submitted work. The authors declare that they do not have any other conflicts of interest related to this work.

\section{References}

1. Condes E, Arribas JR; COVID19 MADRID-S.P.P.M. group. Impact of COVID-19 on Madrid hospital system. Enferm Infecc Microbiol Clin. 2020. doi:10.1016/j.eimc.2020.06.005
2. Dafer RM, Osteraas ND, Biller J. Acute stroke care in the coronavirus disease 2019 pandemic. J Stroke Cerebrovasc Dis. 2020;29 (7):104881. doi:10.1016/j.jstrokecerebrovasdis.2020.104881

3. Al-Shamsi HO, Alhazzani W, Alhuraiji A, et al. A practical approach to the management of cancer patients during the novel coronavirus disease 2019 (COVID-19) pandemic: an international collaborative group. Oncologist. 2020;25(6). doi:10.1634/theoncologist.2020-0213.

4. Yu L. Standards and follow-up plan for COVID-19 patients. In: Yu L, editor. Handbook of COVID-19 Prevention and Treatment. Zhejiang University School of Medicine, 2020:45-47.

5. Richardson S, Hirsch JS, Narasimhan M, et al. Presenting characteristics, comorbidities, and outcomes among 5700 patients hospitalized with COVID-19 in the New York City area. JAMA. 2020;323 (20):2052. doi:10.1001/jama.2020.6775

6. Wang $\mathrm{X}, \mathrm{Xu} \mathrm{H}$, Jiang $\mathrm{H}$, et al. The clinical features and outcomes of discharged coronavirus disease 2019 patients: a prospective Cohort study. QJM. 2020;113(9):657-665. doi:10.1093/qjmed/hcaa178

7. Sulaiman S, Richter F, Fuster V, et al. Characterization of patients who return to hospital following discharge from hospitalization for COVID-19. J Gen Intern Med. 2020.

8. Real-Time RT-PCR Panel for Detection 2019-Novel Coronavirus Centers for Disease Control and Prevention, Respiratory Viruses Branch, Division of Viral Diseases. Available from: https://www. who.int/docs/default-source/coronaviruse/uscdcrt-pcr-panel-fordetection-instructions.pdf?sfvrsn=3aa07934_2. Accessed November 19, 2020.

9. Zapatero A, Barba R, Marco J, et al. Predictive model of readmission to internal medicine wards. Eur J Intern Med. 2012;23:451-456. doi:10.1016/j.ejim.2012.01.005

10. Han Y, Jiang M, Xia D, et al. COVID-19 in a patient with long-term use of glucocorticoids: a study of a familial cluster. Clin Immunol. 2020;214:108413. doi:10.1016/j.clim.2020.108413

11. Spezzani V, Piunno A, Iselin HU. Benign COVID-19 in an immunocompromised cancer patient - the case of a married couple. SwissMedWkly. 2020;150:w20246.

12. Fernández-Ruiz M, Andrés A, Loinaz C, et al. COVID-19 in solid organ transplant recipients: a single-center case series from Spain. Am J Transplant. 2020;20(7):1849-1858. doi:10.1111/ajt.15929

13. Huang C, Wang Y, Li X, et al. Clinical features of patients infected with 2019 novel coronavirus in Wuhan, China. Lancet. 2020;395 (10223):497. doi:10.1016/S0140-6736(20)30183-5

14. Cavagna L, Bruno R, Zanframundo G, et al. Clinical presentation and evolution of COVID-19 in immunosuppressed patients. Preliminary evaluation in a North Italian cohort on calcineurin-inhibitors based therapy. MedRxiv. 2020. doi:10.1101/2020.04.26.20080663

15. Tang N, Bai H, Chen X, Gong J, Li D, Sun Z. Anticoagulant treatment is associated with decreased mortality in severe coronavirus disease 2019 patients with coagulopathy. J Thromb Haemost. 2020;18:1094-1099. doi:10.1111/jth.14817

16. Klok FA, Kruip MJHA, van der Meer NJM, et al. Confirmation of the high cumulative incidence of thrombotic complications in critically ill ICU patients with COVID-19: an updated analysis. Thromb Res. 2020.

17. Poggiali E, Bastoni D, Ioannilli E, Vercelli A, Magnacavallo A. Deep vein thrombosis and pulmonary embolism: two complications of COVID-19 pneumonia? Eur J Case Rep Intern Med. 2020;7:001646.

18. Pal R, Banerjee M. Are people with uncontrolled diabetes mellitus at high risk of reinfections with COVID-19? Prim Care Diabetes. 2020. doi:10.1016/j.pcd.2020.08.002

19. Demelo-Rodríguez P, Cervilla-Muñoz E, Ordieres-Ortega L, et al. Incidence of asymptomatic deep vein thrombosis in patients with COVID-19 pneumonia and elevated D-dimer levels. Thromb Res. 2020;192:23-26. doi:10.1016/j.thromres.2020.05.018 


\section{Publish your work in this journal}

The International Journal of General Medicine is an international, peer-reviewed open-access journal that focuses on general and internal medicine, pathogenesis, epidemiology, diagnosis, monitoring and treatment protocols. The journal is characterized by the rapid reporting of reviews, original research and clinical studies across all disease areas. The manuscript management system is completely online and includes a very quick and fair peer-review system, which is all easy to use. Visit http://www.dovepress.com/ testimonials.php to read real quotes from published authors. 